\title{
UTILIZAÇÃO DE PLANTAS MEDICINAIS NA GRAVIDEZ E AMAMENTAÇÃO: REVISÃO INTEGRATIVA
}

\author{
USE OF MEDICINAL PLANTS DURING PREGNANCY AND \\ BREASTFEEDING:INTEGRATIVE REVIEW
}

\author{
Rejanne Lima Arruda ${ }^{1}$ \\ Nathália Olívia de Sousa Garcia ${ }^{2}$ \\ Evilanna Lima Arruda ${ }^{3}$ \\ Edemilson Cardoso da Conceição ${ }^{4}$
}

\begin{abstract}
RESUMO: OBJETIVO: Identificar estudos que relatem sobre o uso de plantas medicinais por mulheres gestantes ou lactantes. MÉTODOS: Foi realizada uma revisãointegrativa $(\mathrm{RI})$ da literatura nacional com abordagem retrospectiva, no período de 2010 a 2020, disponíveis nas bases de dados SciELO, PubMed e Google Acadêmico, utilizando palavras chave: plantas medicinais na gravidez, fitoterápicos na gestação, fitoterapia materna, gestação e amamentação e uso de plantas medicinais. RESULTADOS: Foram selecionados seis estudos, que atenderam aos critérios de inclusão/exclusão, cinco são artigos publicados em periódicos e uma dissertação. Observa-se com os resultados que todas as gestantes analisadas fizeram uso de algum chá de espécie vegetal e que a maior parte não sabiam dos riscos que esse consumo inadequado poderia trazer para o feto. CONCLUSÃO: O uso de plantas medicinais é uma prática comum entre as gestantes e lactantes e devido a capacidade abortiva de algumas espécies deve-se ter conhecimento dos riscos e das quantidades/concentrações seguras antes da utilização.
\end{abstract}

Palavras chave: Medicina alternativa; produtos naturais, lactantes, gravidez.

ABSTRACT: OBJECTIVE: To identify studies reporting on the use of medicinal plants by pregnant or lactating women. METHODS: An integrative review (IR) of the national literature with a retrospective approach was carried out from 2010 to 2020,

\footnotetext{
${ }^{1}$ Doutora em Inovação Farmacêutica pela Faculdade de Farmácia da Universidade Federal de Goiás.

${ }^{2}$ Doutora em Inovação Farmacêutica pela Faculdade de Farmácia da Universidade Federal de Goiás.

${ }^{3}$ Aluna de Doutorado do Programa de Pós graduação em Inovação Farmacêutica da Faculdade de Farmácia da Universidade Federal de Goiás.

${ }^{4}$ Professor Titular na Faculdade de Farmácia da Universidade Federal de Goiás.
} 
available in the SciELO, PubMed and Google Scholar databases, using keywords: medicinal plants during pregnancy, herbal medicines during pregnancy, maternal phytotherapy, pregnancy and breastfeeding and use of medicinal plants. RESULTS: Six studies were selected, which met the inclusion/exclusion criteria, five are articles published in journals and one dissertation. It is observed with the results that all the pregnant women analyzed made use of some tea of plant species and that most did not know the risks that this inadequate consumption could bring to the fetus. CONCLUSION: The use of medicinal plants is a common practice among pregnant and lactating women and due to theabortive capacity of some species, one should be aware of the risks and safe amounts/concentrations before use.

Keywords: Alternative medicine; natural products, lactating, pregnancy. 


\section{INTRODUÇÃO}

A prática da utilização de plantas medicinais para amenizar sintomas tem sido descrita por praticamente todos os povos. A Organização Mundial de Saúde (OMS) afirma que cerca de65 a $80 \%$ da população mundial não têm acesso ao atendimento primário de saúde e recorre à medicina tradicional, especialmente às plantas medicinais, na procura de alívio para muitas doenças (LIMA, 2001).

Gestantes e lactantes constituem um grupo populacional que culturalmente recorre ao uso de plantas medicinais, por acreditarem que não causam danos ao concepto. Durante esse período da vida, as mulheres acabam fazendo uso de algumas espécies vegetais sem o real conhecimento dos danos que isso pode causar. De acordo com Maciel (2002) todo medicamento assim como as plantas medicinais devem ser utilizadas de forma racional, pois se forem mal utilizadas podem causar sequelas, aborto e intoxicações.

Deve-se levar em consideração que a resposta farmacológica após a administração de qualquer tipo de substância é distinta ao se relacionar o corpo materno com o corpo fetal. A barreira placentária não é eficaz para conter a maioria dos fármacos e à medida que estes entram em contato com o feto, o efeito vai depender da capacidade do organismo deste em metaboliza-lo e da concentração da substância que irão chegar aos tecidos fetais (GOMES et al, 1999; MAEDA; SECOLI, 2008).

Dentre as plantas medicinais que podem causar riscos na gestação, principalmente se consumidos no primeiro trimestre temos: aloe (Aloe ferox), angélica (Angelica archangelica), eucalipto (Eucaliptus globulus), alecrim (Rosmarinus officinalis), gengibre (Zengiberofficinalis) e sene (Cassia angustifólia e Cassia acutifólia) (DAVIS, 1996).

Em relação a amamentação sabe-se que é algo primordial nos primeiros meses de vida da criança, pois o leite materno fornece as necessidades nutricionais do lactente e auxilia no seusistema imunológico e cognitivo (CAMINHA et al, 2010). 
uso de plantas medicinais pela mãe neste período deve ser feito com cautela pois ela pode transferir metabólitos para a criança a partir do leite materno e causar problemas de saúde ao recém-nascido.

Algumas plantas foram relatadas em estudos devido seu efeito calmante quando utilizados por lactantes, como a Cymbopogon citratus, conhecida como capim santo. Tais efeitos são descritos no Formulário de Fitoterápicos da Farmacopeia Brasileira (BRASIL, 2018) Apesar dos conhecimentos acerca da utilização de medicamentos e outras substâncias durante o período da amamentação tenha sido muito ampliado, ainda é incerto os perigos causados por muitas das substâncias utilizadas pela nutriz, no lactente (BRASIL, 2015).

Diante da importância do tema e da necessidade de ampliar os estudos de modo a esclarecer os riscos e benéficos dessa prática entre as gestantes e lactantes, o objetivo do presente trabalho foi realizar uma revisão de literatura sobre o uso de plantas medicinais entre gestantes e lactantes.

\section{METODOLOGIA}

Foi realizado uma revisão integrativa da literatura que teve por finalidade reunir o conhecimento pré-existente sobre a temática do estudo. O estudo foi realizado em Janeiro de 2021 através da leitura de artigos científicos, por meio de busca on-line nas bases de dados SciELO, PubMed e Google Acadêmico. A questão norteadora da pesquisa foi: Quais os principais grupos de plantas medicinais ou medicamentos fitoterápicos utilizados por mulheres grávidas e lactantes? A partir desse questionamento foram selecionados os materiais utilizandoas palavras chave: plantas medicinais na gravidez, fitoterápicos na gestação, fitoterapia materna, gestação e amamentação e uso de plantas medicinais, as quais seus sinônimos estão nos descritores em Ciências da Saúde (DeSC).

Os critérios de inclusão foram: trabalhos publicados em português ou espanhol entre os anos de 2010 a 2020. Os critérios de exclusão foram: artigos publicados em inglês e que não continham respostas para a pergunta central, textos 
incompletos ou com acesso restrito. Complementou-se o estudo com dissertações de pós graduação.

Posteriormente, foi construído um instrumento para integração dos achados em forma de quadro de modo a dar visibilidade às principais características de cada produção (título, autor/ano de publicação, objetivo e principais resultados), mantendo-se a autenticidade das ideias, conceito e definições dos autores.

\section{RESULTADOS}

Após a fase de seleção dos artigos, foram incluídos na revisão integrativa 6 (seis) estudos, sendo 5 (cinco) artigos científicos e 1 (uma) dissertação de mestrado (Quadro 1) que atenderam aos critérios de inclusão/exclusão previamente estabelecidos e que apresentaram uma resposta a questão norteadora.

Quadro 1: Descrição dos artigos selecionados conforme título, autor/ano de publicação, objetivos e principais resultados.

\begin{tabular}{|c|c|c|c|}
\hline TÍTULO & AUTOR & OBJETIVO & PRINCIPAIS RESULTADOS \\
\hline $\begin{array}{llr}\text { Utilização } & & \text { de } \\
\text { medicamentos } & \text { e } \\
\text { plantas } & \text { medicinais } \\
\text { por } & & \text { gestantes } \\
\text { atendidas } & & \text { na } \\
\text { unidade } & \text { de } & \text { saúde } \\
\text { da } & \text { mulher } & \text { em } \\
\text { Alegre, } & \text { ES, } & \text { Brasil }\end{array}$ & $\begin{array}{l} \\
\\
\text { ZAMPIROLLI } \\
\text { al, } 2017 . \\
\end{array}$ & $\begin{array}{l}\text { Identificar a utilização de } \\
\text { medicamentos e plantas } \\
\text { medicinais junto às gestantes } \\
\text { atendidas na Unidade Saúde } \\
\text { da Mulher, no município de } \\
\text { Alegre, ES. }\end{array}$ & $\begin{array}{l}\text { Os resultados apresentaram que } \\
17 \% \text { das mulheres afirmaram } \\
\text { utilizar no mínimo um tipo de chá } \\
\text { durante o período gestacional, e o } \\
\text { mais utilizado foi o de capim- } \\
\text { cidreira. }\end{array}$ \\
\hline $\begin{array}{l}\text { Percepção de risco } \\
\text { e conceitos obre } \\
\text { plantas medicinais, } \\
\text { fitoterápicos } \\
\text { medicamentos } \\
\text { alopáticos entre } \\
\text { gestantes }\end{array}$ & $\begin{array}{l}\text { PIRES; } \\
\text { ARAÚJO,201 }\end{array}$ & \begin{tabular}{|l} 
Analisar as percepções de \\
risco relacionadas ao uso de \\
fitoterápicos, \\
medicinais e medicamentos \\
alopáticos em gestantes \\
atendidas em uma unidade \\
básica de saúde, bem como \\
apresentar os conceitos \\
definidos por elas a respeito \\
dessesagentes terapêuticos
\end{tabular} & $\begin{array}{l}\text { Após a entrevista com as nove } \\
\text { gestantes, as principais plantas que } \\
\text { as mesmas relataram consumofora: } \\
\text { Capim-santo, capim-cidreira ou } \\
\text { capim-limão (Cynbopogon citratus) } \\
\text { Espinho Cheiroso Kanthoxilum } \\
\text { shifolium / Zanthoxylum Nemorale e } \\
\text { Boldo-da-Bahia, Alumã Vernonia } \\
\text { bahiensi s }\end{array}$ \\
\hline
\end{tabular}




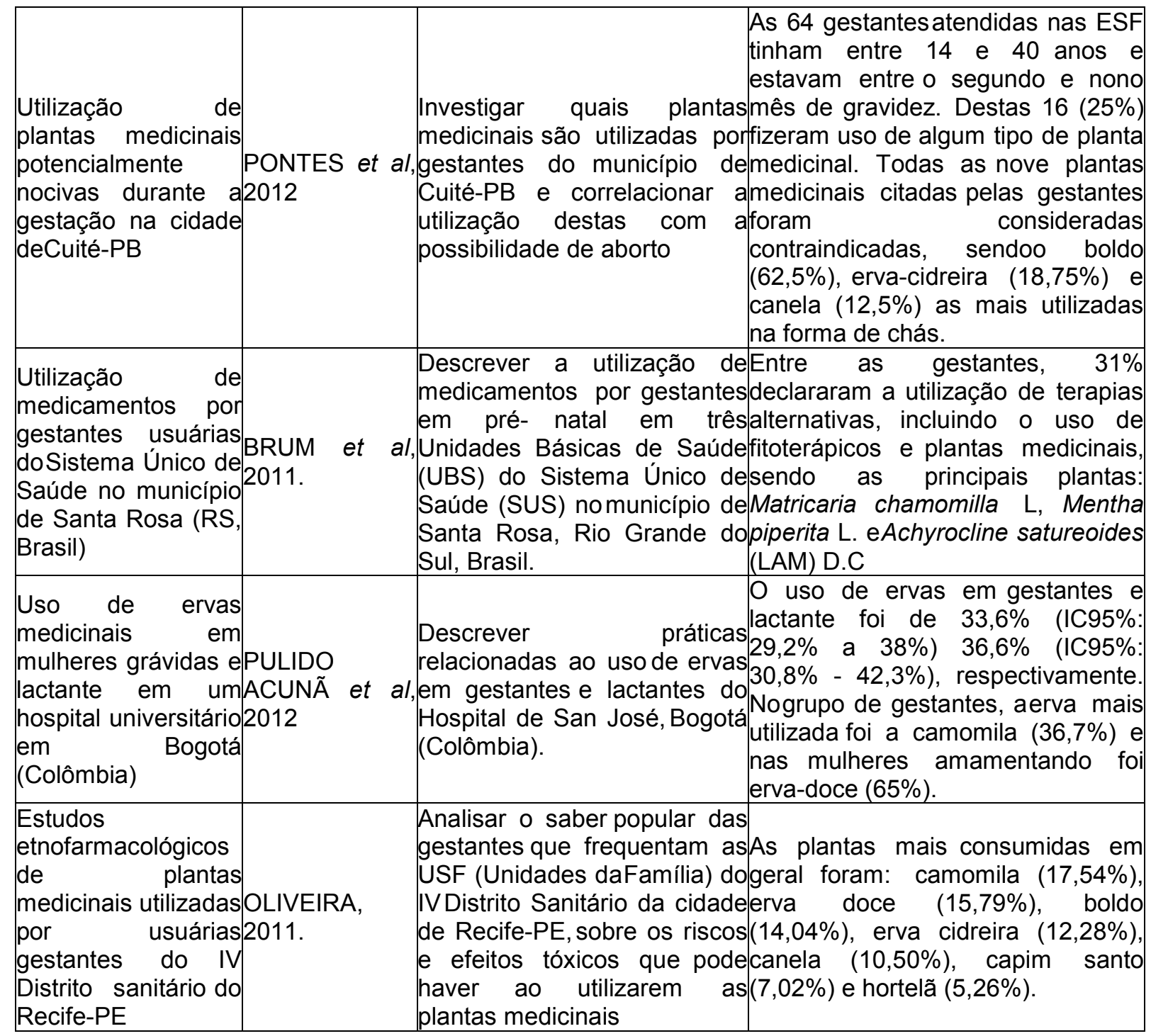

\section{DISCUSSÃo}

O uso de medicamentos sem prescrição médica pode levar a sérias consequências. Como a automedicação é um problema universal, as gestantes também estão sujeitas a essa prática podendo gerar sérias complicações ao feto. Os estudos trazem algumas explicações para essa prática, como a decepção com os tratamentos convencionais, como seus efeitos colaterais, a falta de acesso ao atendimento médico em algumas regiões e/ou a percepção que as plantas medicinais não podem causar risco à saúde. No estudo de Pires e Araújo (2011) 
cinco das novegestantes avaliadas, justificaram o uso de plantas medicinais devido ao rápido efeito de ação, que segundo elas sobressaem aos dos medicamentos alopáticos.

No estudo de Pontes et al (2012) o boldo foi a planta medicinal mais consumida na forma de chá pelas gestantes $(62,5 \%)$, seguido pela erva cidreira $(18,75 \%)$, sendo que estas relataram não ter conhecimento que algumas plantas são capazes de provocar relaxamento do útero, menstruação e até aborto. Ernest (2002) afirma que o uso de qualquer substância, mesmo uma planta medicinal, durante a gravidez deve ser feita com cuidado e com acompanhamento médico especializado.

O estudo de Bergsten-Mendes (2002) relata que o boldo está em quarto lugar na escala de plantas mais consumidas por gestantes, e afirma que a mesma pode levar ao aborto, devendo portanto ser evitado durante o primeiro trimestre de gestação.

Em contrapartida o estudo de Pulido acunã et al (2012) aponta que a camomila (Matricaria chamomilla L) foi a planta mais consumida pelas gestantes no primeiro trimestre de gestação, sendo mais usadas para alívio de cólica e como tranquilizante. Pelas lactantes a planta mais consumida foi a erva doce (Pimpinella anisum) com o intuito de aumentar a produção de leite.

A erva-doce é capaz de causar sedação leve quando utilizada na forma de chá, e associada a fármacos hipnóticos pode prolongar a ação do medicamento. Já a Matricaria chamomilla $L$ pode interagir com anticoagulantes e barbitúricos. Essa espécie é capaz de aumentar os riscos de sangramento quando utilizada com anticoagulantes, como a varfarina; e quando utilizada com barbitúricos, é capaz de atuar em sinergismo com o fármaco, prolongando o efeito depressor do sistema nervoso central (Nicoletti, 2007).

Da mesma forma no estudo de Brum et al (2011) realizado com gestantes que realizarampré-natal nos meses de julho e agosto de 2005, em três das onze UBS no município de Santa Rosa-RS e o de Oliveira (2011) realizado em Recife-PE, mostraram que a Matricaria chamomilla $L$ também foi a planta mais consumida entre as mulheres gestantes avaliadas.

Além das espécies supracitadas temos outras plantas que também fazem parte do arsenalterapêutico de algumas gestantes, como: camomila, o gengibre, a menta, 
a equinácea, o alho, o "cranberry", o aloe, o "raspberry", o "primrose" e o ginseng. Fato curioso que ginseng é uma erva da medicina tradicional chinesa e na pesquisa realizada por Kenned et al (2013) apenas 20\% das gestantes asiáticas relataram fazer uso. As espécies Fenugreek e Trigonella foenum- graecum L. (Fabaceae), foram citadas pelas gestantes do Oriente Médio e do Egito. Nesse mesmo estudo os autores relataram que o gengibre, a menta e o alho foram mencionados por gestantes nos cinco continentes, demonstrando que essas espécies vegetais possuem uso medicinal disseminado em todo o mundo. Outra planta, a camomila, também foi citada como erva utilizada por gestantes de todos os continentes, exceto 0 africano.

Em se tratando das vias de administração utilizadas pelas gestantes para o consumo dasplantas medicinais, estas podem costumam ser oral, na forma de chás (decocção/infusão) $(86,45 \%)$, lambedores $(9,35 \%)$ e outras formas como tópicas e banhos (6,07\%) (OLIVEIRA, 2011). Já em relação a frequência de uso, no estudo de Oliveira (2011) as gestantes relataram utilizar duas vezes ao dia (29,37\%), uma vez ao dia $(26,57 \%)$, três vezes ao dia $(26,57 \%)$ e acima de três vezes ao dia $(17,49 \%)$. Algumas gestantes relataram as reações adversas como sangramento, vômitos e diarreias, após ingestão de preparações a base de quebra pedra (Phylllantus sp), losna (Artemisia absinthium), cabacinho (Luffa operculata), boldo (Peumus boldus), caqueja (Baccharis crispa Spreng), chá verde (Camellia sinensis L), sena (Senna alexandrina Miller) e espirradeira (Nerium oleander).

Alguns estudos apontam o uso consciente de plantas medicinais com capacidade abortiva por mulheres gestantes. O estudo de Moreira et al (2001) constatou que as ervas maisfrequentes usadas com objetivo da ação abortiva foram: Alumã (Vermonia baiensis), espinho-cheiroso (Xanthoxilium rhoifolium).

Quina verdadeira (Cinchona calysaya), Hortelã (Mentha pulegium), Romã (Punica granatum), Quitoco (Pluchea quitoco) e Arruda (Ruta graveolens L). Sabe-se que a utilização de chás feitos de plantas medicinais como prática abortiva, constitui uma prática ilegal no Brasil e é considerado um grande problema de saúde pública (CARVALHO et al, 2003).

Com relação ao uso por lactantes, algumas mães relatam fazer uso de plantas medicinais que apresentam na cultura popular efeito galactagogo, como por 
exemplo: Trigonella (FABACEAE), conhecida como feno grego e a Foeniculum vulgare, conhecida como funcho (BADGUJAR et al, 2014).

Além dessas, a planta medicinal conhecida como quebra pedra (Phyllanthus niruri) também é bastante utilizada por lactantes e pode ser responsável por causar cólicas e diarreias no recém-nascido (OLIVEIRA, 2011).

Com base nos achados é possível analisar que o uso de plantas medicinais é algo comum pela população, sobretudo nas mulheres grávidas e lactantes, e que deve ser conscientizada de modo a ser utilizado com acompanhamento e após um estudo prévio sobre as possíveis consequências das substâncias presentes para a saúde materno-infantil.

\section{CONCLUSÃO}

Diante disso, afirma-se que a utilização de chás durante a gravidez tem suas restrições, uma vez que dada a variabilidade individual, algumas gestantes podem ser mais sensíveis a menores concentrações do produto vegetal utilizado, ou seja, o grau de toxidade depende da dosagem e da fisiologia do organismo analisado. Essa prática da automedicação com plantas medicinais pode causar sérios riscos à saúde da mãe e da criança e por isso não deve ser propagada. 


\section{REFERÊNCIAS BIBLIOGRÁFICAS}

BADGUJAR, S.B; PATEL, V.V; BANDIVDEKAR, A.H. Foeniculum vulgare Mill: a review of its botany, phytochemistry, pharmacology, contemporary apllication, and toxicology. BioMed. Res. Int. p.1-32, 2014.

BERGSTEN-MENDES, G. Prevalência do uso de medicamentos na gravidez: uma abordagem farmacoepidemiológica. Revista Saúde Pública, 36(2):205-212, 2002.

BORGES, R.A.M.; OLIVERIA, V. Riscos Associados ao Uso de Plantas Medicinais Durante o Período da Gestação: Uma Revisão. Revista UNIANDRADE. 16. 101-108, 2015.

BRASIL. Ministério da Saúde. Secretaria de Atenção à Saúde. Departamento de Atenção Básica. Saúde da criança: nutrição infantil - Aleitamento materno e alimentação complementar. [Normas e Manuais Técnicos. $2^{\mathrm{a}}$ Edição - Caderno de Atenção Básica ${ }^{\circ}{ }^{\circ} 23$ ]. Brasília: Ministério da Saúde, 2015.

BRASIL. Agência Nacional de Vigilância Sanitária. Formulário de Fitoterápicos da Farmacopéia Brasileira. Brasília: Anvisa, p. 160, 2018.

BRUM, L.F.S, PEREIRA, P; FELICETTI, L.L, SILVEIRA, R.D. Utilização de medicamentos por gestantes usuárias do Sistema Único de Saúde no município de Santa Rosa (RS, Brasil). Ciênc. saúde coletiva, v,16 ( 5 ): 2435-2442, 2011.

CAMINHA, M.F.C.; SERVA, V.B.; ARRUDA, I.K.; FILHO, M.B. Aspectos históricos, socioeconômicos e institucionais do aleitamento materno. Revista Brasileira de Saúde Materno infantil, v.10, n.1, p.25-37, 2010.

CARVALHO, AG; GOMES, JAL; LEMOS VM; OLIVEIRA, TMF; FONSECA, BTS, ESTEVES, APVS. Consequências fisiopatológicas ao uso de abortifacientes durante a gestação. Revista PUC, 2003.

DAVIS, P. Aromaterapia. Ed. Martins Fontes: São Paulo, 1996.

ERNST, E. - Herbal medicinal products during pregnancy: are they safe? International. J. Obstet. Gynaecol. 109: 227-235, 2002.

GOMES, KR; MORON AF; SILVA RS; SIQUEIRA AA. Prevalência do uso de medicamentosna gravidez e relações com as características maternas. Rev. Saúde Pública. V. 33(3): 246- 254, 1999.

KENNEDY DA; LUPATTELLI A; KOREN G; NORDENG $H$. Herbal medicine use in pregnancy: results of a multinational. BMC Complement Altern Med, v.13:355, 2013.

MACIEL, M.A; PINTO, A.C; VEIGA JR, V.F. Plantas medicinais: a necessidade de estudos multidisciplinares. Quimica Nova.v.25, n.3, 2002.

MAEDA ST, SECOLI SR. Utilização e custo de medicamentos em gestantes de baixo-risco.

Revista Latino-Am. Enfermagem. v.16(2): 266-271, 2008.

MOREIRA, L; DIAS, A.L.D; RIBEIRO, H.B.S. Associação entre uso de abortifacientes e defeitos congênitos. RBGO. v.23, n.8, p.517-521, 2001.

NICOLETTI MA; OLIVEIRA-JUNIOR, MA, BERTASSO, CC, COPOROSSI, PY, TAVARES 
APL. Principais interações no uso de medicamentos fitoterápicos. Infarma, v. 19(1): 32-40, 2007.

PIRES, ANDREA; ARAÚJO, PATRÍCIA. PERCEPÇÃO DE RISCO E CONCEITOS SOBRE PLANTAS MEDICINAIS, FITOTERÁPICOS E MEDICAMENTOS ALOPÁTICOS ENTRE GESTANTES. Revista Baiana de Saúde Pública. 35, 2012.

PONTES, SM; SOUZA, APM; BARRETO, BF; OLIVEIRA, HSB; OLIVEIRA, LBP; SARAIVA, AM; COSTA DA, CARMO ES. Utilização de plantas medicinais potencialmente nocivas durante a gestação na cidade de Cuité-PB. Com. Ciências Saúde, v 23(4):305-311, 2012.

PULIDO ACUÑA, G.P; VÁSQUEZ SEPÚLVEDA, P.L.M, VILLAMIZAR, G.L. Uso de hierbas medicinales en mujeres gestantes y en lactancia en un hospital universitario de Bogotá (Colombia). Index Enferm. 21( 4 ): 199-203, 2012.

RODRIGUES DE OLIVEIRA, Jenifer. Estudo etnofarmacológico de plantas medicinais utilizadas por usuárias gestantes do IV Distrito Sanitário do Recife PE. 2011. Master's Thesis. Universidade Federal de Pernambuco.

ZAMPIROLLI, A.C.D et al. UTILIZAÇÃO DE MEDICAMENTOS E PLANTAS MEDICINAIS POR GESTANTES ATENDIDAS NA UNIDADE DE SAÚDE DA MULHER EM ALEGRE, ES, BRASIL. Infarma - Ciências Farmacêuticas,[S.I.], v. 29, n. 4, p. 349-356,dec. 2017. 